PROCEEDINGS OF THE

AMERICAN MATHEMATICAL SOCIETY

Volume 131, Number 4, Pages 1031-1041

S 0002-9939(02)06620-0

Article electronically published on August 28, 2002

\title{
ON FUNCTIONS WHOSE GRAPH IS A HAMEL BASIS
}

\author{
KRZYSZTOF PŁOTKA
}

(Communicated by Alan Dow)

\begin{abstract}
We say that a function $h: \mathbb{R} \rightarrow \mathbb{R}$ is a Hamel function $(h \in \mathrm{HF})$ if $h$, considered as a subset of $\mathbb{R}^{2}$, is a Hamel basis for $\mathbb{R}^{2}$. We prove that every function from $\mathbb{R}$ into $\mathbb{R}$ can be represented as a pointwise sum of two Hamel functions. The latter is equivalent to the statement: for all $f_{1}, f_{2} \in \mathbb{R}^{\mathbb{R}}$ there is a $g \in \mathbb{R}^{\mathbb{R}}$ such that $g+f_{1}, g+f_{2} \in$ HF. We show that this fails for infinitely many functions.
\end{abstract}

\section{INTRODUCTION}

The terminology is standard and follows 2]. The symbols $\mathbb{R}$ and $\mathbb{Q}$ stand for the sets of all real and all rational numbers, respectively. A basis of $\mathbb{R}^{n}$ as a linear space over $\mathbb{Q}$ is called a Hamel basis. For $Y \subset \mathbb{R}^{n}$, the $\operatorname{symbol}^{\operatorname{Lin}} \mathbb{Q}(Y)$ stands for the smallest linear subspace of $\mathbb{R}^{n}$ over $\mathbb{Q}$ that contains $Y$. The zero element of $\mathbb{R}^{n}$ is denoted by 0 . The cardinality of a set $X$ we denote by $|X|$. In particular, $\mathfrak{c}$ stands for $|\mathbb{R}|$. Given a cardinal $\kappa$, we let $\operatorname{cf}(\kappa)$ denote the cofinality of $\kappa$. We say that a cardinal $\kappa$ is regular if $\operatorname{cf}(\kappa)=\kappa$. For any set $X$, the symbol $[X]^{<\kappa}$ denotes the set $\{Z \subseteq X:|Z|<\kappa\}$. For $A, B \subseteq \mathbb{R}^{n}, A+B$ stands for $\{a+b: a \in A, b \in B\}$.

We consider only real-valued functions. No distinction is made between a function and its graph. For any two partial real functions $f, g$ we write $f+g, f-g$ for the sum and difference functions defined on $\operatorname{dom}(f) \cap \operatorname{dom}(g)$. The class of all functions from a set $X$ into a set $Y$ is denoted by $Y^{X}$. We write $f \mid A$ for the restriction of $f \in Y^{X}$ to the set $A \subseteq X$. For $B \subseteq \mathbb{R}^{n}$ its characteristic function is denoted by $\chi_{B}$. For any function $g \in \mathbb{R}^{X}$ and any family of functions $F \subseteq \mathbb{R}^{X}$ we define $g+F=\{g+f: f \in F\}$. For any planar set $P$, we denote its $x$-projection by $\operatorname{dom}(P)$.

The cardinal function $\mathrm{A}(\mathrm{F})$, for $\mathrm{F} \varsubsetneqq \mathbb{R}^{X}$, is defined as the smallest cardinality of a family $G \subseteq \mathbb{R}^{X}$ for which there is no $g \in \mathbb{R}^{X}$ such that $g+G \subseteq$ F. It was investigated for many different classes of real functions; see e.g., 4, 5, 10. Recall here that $\mathrm{A}(\mathrm{F}) \geq 3$ is equivalent to $\mathrm{F}-\mathrm{F}=\mathbb{R}^{X}$ (see [12, Proposition 1]).

One of the very important concepts in Real Analysis is additivity. It dates back to the early 19th century when the following functional equation was considered

Received by the editors September 13, 2001 and, in revised form, November 2, 2001.

2000 Mathematics Subject Classification. Primary 15A03, 54C40; Secondary 26A21, 54C30.

Key words and phrases. Hamel basis, additive functions, Hamel functions.

Most of this work was done when the author was working on his Ph.D. at West Virginia University. The author wishes to thank his advisor, Professor K. Ciesielski, for many helpful conversations. 
for the first time:

$$
f(x+y)=f(x)+f(y) \text { for all } x, y \in \mathbb{R} .
$$

An obvious solution to this equation is a linear function, that is, a function defined by $f(x)=a x$ for all $x \in \mathbb{R}$, where $a$ is some constant. The fact that the linear functions are the only continuous solutions, was first proved by A. L. Cauchy [1]. Because of this, the above equation is known as Cauchy's Functional Equation. The problem of the existence of a discontinuous solution of the Cauchy equation was solved by G. Hamel in 1905 [6] who constructed a discontinuous function which satisfies the desired equation. To construct an example of such a function observe first that $f \in \mathbb{R}^{\mathbb{R}}$ satisfies the Cauchy equation if and only if it is linear over $\mathbb{Q}$, i.e., for all $p, q \in \mathbb{Q}$ and $x, y \in \mathbb{R}$ we have $f(p x+q y)=p f(x)+q f(y)$. So to define an additive function it is enough to define it on a Hamel basis. Thus, if $H \subseteq \mathbb{R}$ is a Hamel basis and $f$ identically equals 1 on $H$, then clearly $f$ is not continuous. The family of all solutions of Cauchy's Functional Equation is called the family of additive functions and we denote it by AD. For the class of additive functions defined on $\mathbb{R}^{n}$ we use the symbol $\operatorname{AD}\left(\mathbb{R}^{n}\right)$.

It is obvious that not all functions are additive. But one could wonder how "badly" the additive condition can be violated. In particular, does there exist a function $f$ for which the condition $f(x+y)=f(x)+f(y)$ fails for all $x$ and $y$ ? It turns out that the answer is positive. We give two examples of families of such functions. In Section 2 we define and discuss a class of functions whose graph is a linearly independent set over $\mathbb{Q}$. Then, in Section 3 , we investigate a proper subfamily of this class: functions whose graph is a Hamel basis. In this section we state and prove the main result of this paper (Theorem 3.4) which says that every real function is the pointwise sum of two Hamel functions.

\section{FUNCTIONS WITH LINEARLY INDEPENDENT GRAPHS}

Definition 2.1. We say that a function $f: \mathbb{R}^{n} \rightarrow \mathbb{R}$ is linearly independent over $\mathbb{Q}$ (shortly: linearly independent) if $f$ is a linearly independent subset of the space $\left\langle\mathbb{R}^{n+1} ; \mathbb{Q} ;+; \cdot\right\rangle$.

The symbol $\operatorname{LIF}\left(\mathbb{R}^{n}\right)$ stands for the family of all linearly independent functions. In the case when $n=1$, we simply write LIF. An easy example shows that the family $\operatorname{LIF}\left(\mathbb{R}^{n}\right)$ is non-empty for all $n \geq 1$.

Example 2.2. Every injection from $\mathbb{R}^{n}$ into a linearly independent set $H \subseteq \mathbb{R}$ is linearly independent over $\mathbb{Q}$.

Proof. Let $f: \mathbb{R}^{n} \rightarrow H$ be an injection. Assume that for some $p_{1}, \ldots, p_{n} \in$ $\mathbb{Q}$ and pairwise different $x_{1}, \ldots, x_{n} \in \mathbb{R}^{n}$ we have $\sum_{1}^{n} p_{i}\left\langle x_{i}, f\left(x_{i}\right)\right\rangle=0$. Since $f\left(x_{1}\right), \ldots, f\left(x_{n}\right) \in H$ are all different and $H$ is linearly independent over $\mathbb{Q}$, we conclude that $p_{1}=\cdots=p_{n}=0$.

As mentioned in the introductory part of this paper, the linearly independent functions lack the additive property. Thus, $\operatorname{AD}\left(\mathbb{R}^{n}\right) \cap \operatorname{LIF}\left(\mathbb{R}^{n}\right)=\emptyset$.

Below we give some basic properties of the class $\operatorname{LIF}\left(\mathbb{R}^{n}\right)$. Note that Fact 2.3 (i) has its counterpart in the case of continuous and Sierpiński-Zygmund functions (for the definition see [12]). 
Fact 2.3. (i) $\operatorname{LIF}\left(\mathbb{R}^{n}\right)+\operatorname{AD}\left(\mathbb{R}^{n}\right)=\operatorname{LIF}\left(\mathbb{R}^{n}\right)$.

(ii) If $f \in \operatorname{LIF}\left(\mathbb{R}^{n}\right)$, then $\left|f\left[\mathbb{R}^{n}\right]\right|=\mathfrak{c}$.

(iii) If $f: \mathbb{R}^{n} \rightarrow \mathbb{R}$ is continuous on a non-empty open set, then $f \notin \operatorname{LIF}\left(\mathbb{R}^{n}\right)$.

(iv) There exists an $f \in \operatorname{LIF}\left(\mathbb{R}^{n}\right)$ which is the union of countably many partial continuous functions.

(v) $\mathrm{A}\left(\operatorname{LIF}\left(\mathbb{R}^{n}\right)\right)=\mathfrak{c}$.

Proof. (i) Let $f \in \operatorname{LIF}\left(\mathbb{R}^{n}\right)$ and $g \in \mathrm{AD}\left(\mathbb{R}^{n}\right)$. Fix $x_{1}, \ldots, x_{k} \in \mathbb{R}^{n}$ and $q_{1}, \ldots, q_{k} \in$ $\mathbb{Q}$. Now suppose that $\sum_{1}^{k} q_{i}\left\langle x_{i}, f\left(x_{i}\right)+g\left(x_{i}\right)\right\rangle=0$. Thus, in particular, $\sum_{1}^{k} q_{i} x_{i}=0$. Since $g$ is additive we have $\sum_{1}^{k} q_{i} g\left(x_{i}\right)=0$. Consequently, $\sum_{1}^{k} q_{i}\left\langle x_{i}, f\left(x_{i}\right)\right\rangle=0$. The linear independence of $f$ implies that $q_{1}=\cdots=q_{k}=0$. So $f+g \in \operatorname{LIF}\left(\mathbb{R}^{n}\right)$.

(ii) Notice that it suffices to prove part (ii) for $n=1$. Assume, by the way of contradiction, that $f \in \operatorname{LIF}$ and $|f[\mathbb{R}]|=\kappa<\mathfrak{c}$. We claim that there exist $x_{1}, x_{2} \in \mathbb{R}$ with the following properties:

$$
x_{1} \neq x_{2}, f\left(x_{1}\right)=f\left(x_{2}\right) \text {, and } f\left(-x_{1}\right)=f\left(-x_{2}\right) .
$$

To see the claim choose $y_{0} \in \mathbb{R}$ such that $\left|f^{-1}\left(y_{0}\right) \cap(0, \infty)\right| \geq \kappa^{+}$. Such an element exists because $(0, \infty) \subseteq \bigcup_{y \in \mathbb{R}} f^{-1}(y)$ and $|f[\mathbb{R}]|=\kappa<\mathfrak{c}$. Since $y_{0}$ satisfies the condition $\left|f\left[-f^{-1}\left(y_{0}\right)\right]\right| \leq \kappa<\kappa^{+} \leq\left|-f^{-1}\left(y_{0}\right)\right|$, there exist different $x_{1}, x_{2} \in$ $f^{-1}\left(y_{0}\right) \cap(0, \infty)$ satisfying the equality $f\left(-x_{1}\right)=f\left(-x_{2}\right)$. Note that $x_{1}$ and $x_{2}$ are the required points. Next observe that

$$
\left\langle x_{1}, f\left(x_{1}\right)\right\rangle+\left\langle-x_{1}, f\left(-x_{1}\right)\right\rangle=\left\langle x_{2}, f\left(x_{2}\right)\right\rangle+\left\langle-x_{2}, f\left(-x_{2}\right)\right\rangle .
$$

This leads to a contradiction with $f \in$ LIF.

(iii) As in part (ii), it is enough to prove the case $n=1$. Let $(a-h, a+h) \subseteq \mathbb{R}$ be a non-empty open interval such that $f \mid(a-h, a+h)$ is continuous. Consider a function $g:[0, h) \rightarrow \mathbb{R}$ defined by $g(x)=f(a-x)+f(a+x)$. Obviously, $g$ is also continuous. If $g(x)=g(0)=2 f(a)$ for all $x \in[0, h)$, then $f$ is not linearly independent. Hence we may suppose that there exist two different $x_{1}, x_{2} \in(0, h)$ such that $g\left(x_{1}\right)=2 f(a)+p_{1}$ and $g\left(x_{2}\right)=2 f(a)+p_{2}$ for some non-zero rationals $p_{1}, p_{2}$. Then we have

$$
p_{2}\left\langle 2 a, g\left(x_{1}\right)\right\rangle-p_{1}\left\langle 2 a, g\left(x_{2}\right)\right\rangle \in \operatorname{Lin}_{\mathbb{Q}}(\langle 2 a, 2 f(a)\rangle)=\operatorname{Lin}_{\mathbb{Q}}(\langle a, f(a)\rangle) .
$$

Now, recall the definition of $g$ and note $\left\langle a-x_{i}, f\left(a-x_{i}\right)\right\rangle+\left\langle a+x_{i}, f\left(a+x_{i}\right)\right\rangle=$ $\left\langle 2 a, g\left(x_{i}\right)\right\rangle$ for $i=1,2$. Based on (2.1), we see that $f$ is not linearly independent.

(iv) Let us first recall that $\mathbb{R}^{n}$ can be decomposed into $(n+1)$ 0-dimensional spaces $E_{0}, \ldots, E_{n}$. For every perfect set $Q \subseteq \mathbb{R}$ and 0-dimensional space $E$ there exists an embedding $h_{Q}^{E}: E \rightarrow Q$. (See e.g., 7].) It is also known that there exists a perfect set $P \subseteq \mathbb{R}$ which is linearly independent over $\mathbb{Q}$. (See e.g., [8].) Now, if $P=P_{0} \cup P_{1} \cup \cdots \cup P_{n}$ is a partition of $P$ into $(n+1)$ perfect sets, then, by Example 2.2 $h_{P_{i}}^{E_{i}}: E_{i} \rightarrow P_{i}(i=0, \ldots, n)$ is a linearly independent subset of $\mathbb{R}^{n+1}$. It is easy to see that $h=\bigcup_{0}^{n} h_{P_{i}}^{E_{i}}: \mathbb{R}^{n} \rightarrow P$ is one-to-one. So, again by Example 2.2 $h$ is linearly independent. Obviously, $h$ is the union of countably many partial continuous functions.

(v) We start by showing that $\mathrm{A}\left(\operatorname{LIF}\left(\mathbb{R}^{n}\right)\right) \geq \mathfrak{c}$. Let $\mathbb{R}^{n}=\left\{x_{\xi}: \xi<\mathfrak{c}\right\}$. Fix an $F \subseteq \mathbb{R}^{\mathbb{R}^{n}}$ of cardinality less than continuum. We will define, by induction, a function $h: \mathbb{R}^{n} \rightarrow \mathbb{R}$ such that for every $f \in F, h+f$ is one-to-one and $(h+f)\left[\mathbb{R}^{n}\right]$ is linearly independent. Then, by Example $2.2 h+F \subseteq \operatorname{LIF}\left(\mathbb{R}^{n}\right)$. 
Let $\alpha<\mathfrak{c}$. Assume that $h$ is defined on $\left\{x_{\xi}: \xi<\alpha\right\}$, for all $f \in F$ the function $h+f$ is one-to-one, and $(h+f)\left[\left\{x_{\xi}: \xi<\alpha\right\}\right]$ is linearly independent. We will define $h\left(x_{\alpha}\right)$. Choose

$$
h\left(x_{\alpha}\right) \in \mathbb{R} \backslash \operatorname{Lin}_{\mathbb{Q}}\left(\bigcup_{f \in F}\left((h+f)\left[\left\{x_{\xi}: \xi<\alpha\right\}\right] \cup\left\{f\left(x_{\alpha}\right)\right\}\right)\right) .
$$

This choice is possible since

$$
\left|\bigcup_{f \in F}\left((h+f)\left[\left\{x_{\xi}: \xi<\alpha\right\}\right] \cup\left\{f\left(x_{\alpha}\right)\right\}\right)\right| \leq(\alpha+1)|F|<\mathfrak{c} .
$$

It is easy to see that all the required properties of $h$ are preserved. This ends the proof of $\mathrm{A}\left(\operatorname{LIF}\left(\mathbb{R}^{n}\right)\right) \geq \mathfrak{c}$.

To see the opposite inequality consider $F$ consisting of all constant functions. Then for any function $h: \mathbb{R}^{n} \rightarrow \mathbb{R}$ there is an $f \in F$ such that $h(0)+f(0)=0$. Therefore $h+f \notin \operatorname{LIF}\left(\mathbb{R}^{n}\right)$.

\section{HAMEL FUnCTIONS}

In this section we confine ourselves to a proper subclass of linearly independent functions. More precisely, we consider the class of Hamel functions. We say that a function $f: \mathbb{R}^{n} \rightarrow \mathbb{R}$ is a Hamel function $\left(f \in \operatorname{HF}\left(\mathbb{R}^{n}\right)\right.$ or $f \in \mathrm{HF}$ for $\left.n=1\right)$ if $f$, considered as a subset of $\mathbb{R}^{n+1}$, is a Hamel basis for $\mathbb{R}^{n+1}$. Clearly, $\operatorname{HF}\left(\mathbb{R}^{n}\right) \subseteq$ $\operatorname{LIF}\left(\mathbb{R}^{n}\right)$. A little more challenging argument, comparing it with the case of linearly independent functions, proves the existence of a Hamel function. We do not present it here since this observation is a corollary of Theorem 3.4

Fact 2.3 states some basic properties of the class $\operatorname{LIF}\left(\mathbb{R}^{n}\right)$. It is interesting whether the same statements are true for $\operatorname{HF}\left(\mathbb{R}^{n}\right)$. Since $\operatorname{HF}\left(\mathbb{R}^{n}\right) \subseteq \operatorname{LIF}\left(\mathbb{R}^{n}\right)$, the properties (ii) and (iii) hold trivially. A short additional argument shows that (i) is also true. So we can state

Fact 3.1. (i) $\operatorname{HF}\left(\mathbb{R}^{n}\right)+\mathrm{AD}\left(\mathbb{R}^{n}\right)=\operatorname{HF}\left(\mathbb{R}^{n}\right)$.

(ii) If $f \in \operatorname{HF}\left(\mathbb{R}^{n}\right)$, then $\left|f\left[\mathbb{R}^{n}\right]\right|=\mathfrak{c}$.

(iii) If $f: \mathbb{R}^{n} \rightarrow \mathbb{R}$ is continuous on a non-empty open set, then $f \notin \operatorname{HF}\left(\mathbb{R}^{n}\right)$.

However, it remains an open problem whether Fact 2.3(iv) still holds when $\operatorname{LIF}\left(\mathbb{R}^{n}\right)$ is replaced by $\operatorname{HF}\left(\mathbb{R}^{n}\right)$.

Problem 3.2. Does there exist an $h \in \operatorname{HF}\left(\mathbb{R}^{n}\right)$ which is the union of countably many partial continuous functions?

But it turns out that the statement of the last part of Fact 2.3 is false for the class $\operatorname{HF}\left(\mathbb{R}^{n}\right)$.

Fact 3.3. $\mathrm{A}\left(\operatorname{HF}\left(\mathbb{R}^{n}\right)\right) \leq \omega$ for every $n \geq 1$.

Proof. For each $q \in \mathbb{Q}$ and each open ball $B$ with rational center and radius (rational ball), let us define a function $f_{q}^{B}: \mathbb{R}^{n} \rightarrow \mathbb{R}$ by $f_{q}^{B}=q \chi_{B}$. We claim that for every function $f: \mathbb{R}^{n} \rightarrow \mathbb{R}$ there exist a $q \in \mathbb{Q}$ and a rational ball $B$ such that $f+f_{q}^{B} \notin \operatorname{HF}\left(\mathbb{R}^{n}\right)$. To see this, first note that we may assume that $f=f+f_{0}^{B} \in$ $\operatorname{HF}\left(\mathbb{R}^{n}\right)$. Thus, $\langle 0,1\rangle \in \operatorname{Lin}_{\mathbb{Q}}(f)$. Consequently, there exist $x_{1}, \ldots, x_{k} \in \mathbb{R}^{n}$ and $p_{1}, \ldots, p_{k} \in \mathbb{Q}$ satisfying $\sum_{i=1}^{k} p_{i}\left\langle x_{i}, f\left(x_{i}\right)\right\rangle=\langle 0,1\rangle$. 
Without loss of generality we may assume that $p_{1} \neq 0$. Now let $q=\frac{-1}{p_{1}}$ and $B$ be a rational ball containing $x_{1}$ but not $x_{2}, \ldots, x_{k}$. It follows easily that $f+f_{q}^{B}$ is not linearly independent over $\mathbb{Q}$. Indeed,

$$
\begin{aligned}
\sum_{i=1}^{k} p_{i}\left\langle x_{i}, f\left(x_{i}\right)+f_{q}^{B}\left(x_{i}\right)\right\rangle & =\sum_{i=1}^{k} p_{i}\left\langle x_{i}, f\left(x_{i}\right)\right\rangle+\sum_{i=1}^{k} p_{i}\left\langle 0, f_{q}^{B}\left(x_{i}\right)\right\rangle \\
& =\langle 0,1\rangle+p_{1}\langle 0, q\rangle=0 .
\end{aligned}
$$

Notice here that $\mathrm{A}(\mathrm{LIF})=\mathfrak{c}($ Fact $2.3(\mathrm{v}))$ implies, in particular, that every function from $\mathbb{R}^{\mathbb{R}}$ can be written as the algebraic sum of two linearly independent functions. In other words, LIF $+\mathrm{LIF}=\mathbb{R}^{\mathbb{R}}$. Since we only found the upper bound for $\mathrm{A}(\mathrm{HF})$, it would be very interesting to determine whether $\mathrm{HF}+\mathrm{HF}=\mathbb{R}^{\mathbb{R}}$. The answer to the latter is given in the main result of this paper, Theorem 3.4

Theorem 3.4. Every real function $f: \mathbb{R}^{n} \rightarrow \mathbb{R}$ can be represented as a sum of two Hamel functions. In other words, $\mathbb{R}^{\mathbb{R}^{n}}=\operatorname{HF}\left(\mathbb{R}^{n}\right)+\operatorname{HF}\left(\mathbb{R}^{n}\right)$.

Theorem 3.4 and Fact 3.3 give us the bounds for $\mathrm{A}\left(\operatorname{HF}\left(\mathbb{R}^{n}\right)\right)$. Namely, $3 \leq$ $\mathrm{A}\left(\mathrm{HF}\left(\mathbb{R}^{n}\right)\right) \leq \omega$. It is not known whether the techniques used in the proofs of these two results could also be used to determine $\mathrm{A}\left(\mathrm{HF}\left(\mathbb{R}^{n}\right)\right)$ exactly. We state the next open problem.

Problem 3.5. Does $\mathrm{A}\left(\mathrm{HF}\left(\mathbb{R}^{n}\right)\right)=\omega$ ?

Before proving the theorem we introduce some definitions and show auxiliary results. For $f: \mathbb{R}^{n} \rightarrow \mathbb{R}, x \in \mathbb{R}^{n}$, and $1 \leq k<\omega$ let

$$
\mathrm{LC}(f, k, x)=\left\{\sum_{1}^{k} p_{i} f\left(x_{i}\right): p_{j} \in \mathbb{Q}, x_{j} \in \mathbb{R}^{n}(j=1, \ldots, k), \sum_{1}^{k} p_{i} x_{i}=x\right\} .
$$

When $x=0$ we write $\operatorname{LC}(f, k)$. We also use $\operatorname{LC}(f)$ to denote $\bigcup_{1 \leq k<\omega} \operatorname{LC}(f, k)$. Observe that $\operatorname{LC}(f)$ is a linear subspace of $\mathbb{R}$ over $\mathbb{Q}$, i.e., $\operatorname{LC}(f)=\operatorname{Lin}_{\mathbb{Q}}(\operatorname{LC}(f))$. This is so because $\operatorname{LC}(f)$ is linearly isomorphic to $\operatorname{Lin}_{\mathbb{Q}}(f) \cap(\{0\} \times \mathbb{R})$.

The sets LC $(f)$ will play an important role in the proof of Theorem 3.4. Hence, we will investigate properties of these sets.

Property 3.6. $\mathrm{LC}(f, k) \subseteq \mathrm{LC}(f, 3)+\mathrm{LC}(f, k-1)$ for every $f \in \mathbb{R}^{\mathbb{R}^{n}}$ and $3 \leq k<$ $\omega$.

Proof. Let $y \in \mathrm{LC}(f, k)$. So $y=\sum_{1}^{k} p_{i} f\left(x_{i}\right)$ for some $x_{1}, \ldots, x_{k} \in \mathbb{R}^{n}$ and $p_{1}, \ldots, p_{k} \in \mathbb{Q}$ satisfying $\sum_{1}^{k} p_{i} x_{i}=0$. Define $x^{\prime}=p_{1} x_{1}+p_{2} x_{2}, q=1$, and $r=-1$. Observe that

$$
p_{1} x_{1}+p_{2} x_{2}+r x^{\prime}=q x^{\prime}+p_{3} x_{3}+\cdots+p_{n} x_{k}=0 .
$$

Hence, $p_{1} f\left(x_{1}\right)+p_{2} f\left(x_{2}\right)+r f\left(x^{\prime}\right) \in \mathrm{LC}(f, 3)$ and $q f\left(x^{\prime}\right)+p_{3} f\left(x_{3}\right)+\cdots+$ $p_{n} f\left(x_{k}\right) \in \mathrm{LC}(f, k-1)$. Since $y=p_{1} f\left(x_{1}\right)+p_{2} f\left(x_{2}\right)+r f\left(x^{\prime}\right)+q f\left(x^{\prime}\right)+p_{3} f\left(x_{3}\right)+$ $\cdots+p_{n} f\left(x_{k}\right)$ we conclude that $y \in \mathrm{LC}(f, 3)+\mathrm{LC}(f, k-1)$.

Notice that Property 3.6 implies that

$$
\text { if }|\mathrm{LC}(f)|=\mathfrak{c} \text {, then } m_{0}=\min \{k \geq 1:|\mathrm{LC}(f, k)|=\mathfrak{c}\} \leq 3 .
$$


Next we show another property which is important for the proof of Theorem 3.4 Note that if $\mathfrak{c}$ is regular (i.e., $\operatorname{cf}(\mathfrak{c})=\mathfrak{c}$ ), then the set $Z$ from part (a) can be taken as a singleton.

Property 3.7. Assume that $|\mathrm{LC}(f)|=\mathfrak{c}$. Then at least one of the following two cases hold:

(a) There exists a set $Z \in\left[\mathbb{R}^{n}\right]^{<\mathfrak{c}}$ such that $\left|\bigcup_{z \in Z} \mathrm{LC}(f, 2, z)\right|=\mathfrak{c}$.

(b) For all $X \in\left[\mathbb{R}^{n}\right]^{<\mathfrak{c}}, Y \in[\mathbb{R}]^{<\mathfrak{c}}$ there exist $q_{1}, q_{2}, q_{3} \in \mathbb{Q} \backslash\{0\}$ and pairwise linearly independent $x_{1}, x_{2}, x_{3} \in \mathbb{R}^{n}$ such that $\sum_{1}^{3} q_{i} f\left(x_{i}\right) \notin Y, \sum_{1}^{3} q_{i} x_{i}=0$, and $\operatorname{Lin}_{\mathbb{Q}}\left(x_{1}, x_{2}, x_{3}\right) \cap \operatorname{Lin}_{\mathbb{Q}}(X)=\{0\}$.

Proof. Notice first that if $|\mathrm{LC}(f, 2)|=\mathfrak{c}$, then case (a) holds with $Z=\{0\}$. Hence, using (3.1), we may assume that

$$
|\mathrm{LC}(f, 2)|<\mathfrak{c} \text { and }|\mathrm{LC}(f, 3)|=\mathfrak{c} .
$$

Based on the above assumption and the definition of the set $\mathrm{LC}(f, 3)$, we conclude that there exist continuum many triples $\left\langle x_{1}, x_{2}, x_{3}\right\rangle \in\left(\mathbb{R}^{n}\right)^{3}$ and $\left\langle p_{1}, p_{2}, p_{3}\right\rangle \in$ $(\mathbb{Q} \backslash\{0\})^{3}$ such that $\sum_{1}^{3} p_{i} x_{i}=0$ and $\sum_{1}^{3} p_{i} f\left(x_{i}\right)$ are all different. Thus, an easy cardinal argument implies the existence of a sequence $\left\langle\left\langle x_{1}^{\xi}, x_{2}^{\xi}, x_{3}^{\xi}\right\rangle \in\left(\mathbb{R}^{n}\right)^{3}: \xi<\mathfrak{c}\right\rangle$ and some non-zero rationals $q_{1}, q_{2}, q_{3}$ with the property that $q_{1} x_{1}^{\xi}+q_{2} x_{2}^{\xi}+q_{3} x_{3}^{\xi}=0$ for every $\xi<\mathfrak{c}$, and all $q_{1} f\left(x_{1}^{\xi}\right)+q_{2} f\left(x_{2}^{\xi}\right)+q_{3} f\left(x_{3}^{\xi}\right)$ are different.

Notice that, if $\operatorname{dim}\left(\operatorname{Lin}_{\mathbb{Q}}\left(x_{1}^{\xi}, x_{2}^{\xi}, x_{3}^{\xi}\right)\right)=1$ for some $\xi$, then $\operatorname{Lin}_{\mathbb{Q}}\left(x_{1}^{\xi}, x_{2}^{\xi}, x_{3}^{\xi}\right)=$ $\operatorname{Lin}_{\mathbb{Q}}\left(x_{i}^{\xi}\right)$ for some $i \in\{1,2,3\}$. Say $i=1$. So there is an $s \in \mathbb{Q}$ such that $s q_{1} x_{1}^{\xi}+q_{2} x_{2}^{\xi}=0$. Combining this with the equality $q_{1} x_{1}^{\xi}+q_{2} x_{2}^{\xi}+q_{3} x_{3}^{\xi}=0$ we obtain that $s q_{1} x_{1}^{\xi}+q_{2} x_{2}^{\xi}=(1-s) q_{1} x_{1}^{\xi}+q_{3} x_{3}^{\xi}=0$. Consequently,

$$
\left[s q_{1} f\left(x_{1}^{\xi}\right)+q_{2} f\left(x_{2}^{\xi}\right)\right],\left[(1-s) q_{1} f\left(x_{1}^{\xi}\right)+q_{3} f\left(x_{3}^{\xi}\right)\right] \in \mathrm{LC}(f, 2)
$$

and

$$
\begin{gathered}
q_{1} f\left(x_{1}^{\xi}\right)+q_{2} f\left(x_{2}^{\xi}\right)+q_{3} f\left(x_{3}^{\xi}\right)= \\
s q_{1} f\left(x_{1}^{\xi}\right)+q_{2} f\left(x_{2}^{\xi}\right)+(1-s) q_{1} f\left(x_{1}^{\xi}\right)+q_{3} f\left(x_{3}^{\xi}\right) \in \mathrm{LC}(f, 2)+\mathrm{LC}(f, 2) .
\end{gathered}
$$

So, if $\operatorname{dim}\left(\operatorname{Lin}_{\mathbb{Q}}\left(x_{1}^{\xi}, x_{2}^{\xi}, x_{3}^{\xi}\right)\right)=1$ for continuum many $\xi$, then $|\mathrm{LC}(f, 2)|=\mathfrak{c}$. This contradicts (3.2). Thus, we may assume that $\operatorname{dim}\left(\operatorname{Lin}_{\mathbb{Q}}\left(x_{1}^{\xi}, x_{2}^{\xi}, x_{3}^{\xi}\right)\right)=2$ for all $\xi<\mathfrak{c}$.

Now choose $X \in\left[\mathbb{R}^{n}\right]^{<\mathfrak{c}}$ and $Y \in[\mathbb{R}]^{<\mathfrak{c}}$. Notice that

$(\bullet)$ if $\operatorname{Lin}_{\mathbb{Q}}\left(x_{1}^{\xi}, x_{2}^{\xi}, x_{3}^{\xi}\right) \cap \operatorname{Lin}_{\mathbb{Q}}(X) \neq\{0\}$ and $Z=\operatorname{Lin}_{\mathbb{Q}}(X)$, then

$$
q_{1} f\left(x_{1}^{\xi}\right)+q_{2} f\left(x_{2}^{\xi}\right)+q_{3} f\left(x_{3}^{\xi}\right) \in \bigcup_{z \in Z} \operatorname{LC}(f, 2, z)+\bigcup_{z \in Z} \operatorname{LC}(f, 2, z) .
$$

Indeed, if $\operatorname{Lin}_{\mathbb{Q}}\left(x_{1}^{\xi}, x_{2}^{\xi}, x_{3}^{\xi}\right) \cap \operatorname{Lin}_{\mathbb{Q}}(X) \neq\{0\}$, then there exist $a, b, c \in \mathbb{Q}$ such that $a x_{1}^{\xi}+b x_{2}^{\xi}+c x_{3}^{\xi} \in \operatorname{Lin}_{\mathbb{Q}}(X) \backslash\{0\}$. At least one of the numbers $a, b, c$ is not equal to zero because $a x_{1}^{\xi}+b x_{2}^{\xi}+c x_{3}^{\xi} \neq 0$. Without loss of generality we may suppose that $c \neq 0$ and consequently $c=q_{3}$ (multiply the above equation by $\frac{q_{3}}{c}$ ). Then, by subtracting $a x_{1}^{\xi}+b x_{2}^{\xi}+q_{3} x_{3}^{\xi}$ from $q_{1} x_{1}^{\xi}+q_{2} x_{2}^{\xi}+q_{3} x_{3}^{\xi}=0$, we obtain that $\left(q_{1}-a\right) x_{1}^{\xi}+\left(q_{2}-b\right) x_{2}^{\xi} \in \operatorname{Lin}_{\mathbb{Q}}(X) \backslash\{0\}$. So at least one of $\left(q_{1}-a\right),\left(q_{2}-b\right)$ is not 0 . We may assume that $\left(q_{2}-b\right) \neq 0$. (If $\left(q_{1}-b\right) \neq 0$, then the following argument works analogously.) Now multiply $\left(q_{1}-a\right) x_{1}^{\xi}+\left(q_{2}-b\right) x_{2}^{\xi}$ by $\frac{q_{2}}{q_{2}-b}$. 
We get that $r q_{1} x_{1}^{\xi}+q_{2} x_{2}^{\xi} \in \operatorname{Lin}_{\mathbb{Q}}(X)$ and consequently $(1-r) q_{1} x_{1}^{\xi}+q_{3} x_{3}^{\xi}=$ $\left[q_{1} x_{1}^{\xi}+q_{2} x_{2}^{\xi}+q_{3} x_{3}^{\xi}\right]-\left[r q_{1} x_{1}^{\xi}+q_{2} x_{2}^{\xi}\right]=-\left[r q_{1} x_{1}^{\xi}+q_{2} x_{2}^{\xi}\right] \in \operatorname{Lin}_{\mathbb{Q}}(X)$, for some $r \in \mathbb{Q}$. Hence

$$
\left[r q_{1} f\left(x_{1}^{\xi}\right)+q_{2} f\left(x_{2}^{\xi}\right)\right],\left[(1-r) q_{1} f\left(x_{1}^{\xi}\right)+q_{3} f\left(x_{3}^{\xi}\right)\right] \in \bigcup_{z \in Z} \mathrm{LC}(f, 2, z) .
$$

Now the claim $(\bullet)$ follows from

$$
\begin{aligned}
q_{1} f\left(x_{1}^{\xi}\right)+q_{2} f\left(x_{2}^{\xi}\right)+q_{3} f\left(x_{3}^{\xi}\right) & =r q_{1} f\left(x_{1}^{\xi}\right)+q_{2} f\left(x_{2}^{\xi}\right)+(1-r) q_{1} f\left(x_{1}^{\xi}\right)+q_{3} f\left(x_{3}^{\xi}\right) \\
& \in \bigcup_{z \in Z} \operatorname{LC}(f, 2, z)+\bigcup_{z \in Z} \operatorname{LC}(f, 2, z) .
\end{aligned}
$$

From $(\bullet)$ we see that if $\operatorname{Lin}_{\mathbb{Q}}\left(x_{1}^{\xi}, x_{2}^{\xi}, x_{3}^{\xi}\right) \cap \operatorname{Lin}_{\mathbb{Q}}(X) \neq\{0\}$ holds for $\mathfrak{c}$-many $\xi$, then the set $Z$ satisfies the condition $\left|\bigcup_{z \in Z} \mathrm{LC}(f, 2, z)\right|=\mathfrak{c}$. Obviously $Z \in\left[\mathbb{R}^{n}\right]^{<\mathfrak{c}}$. Thus, case (a) holds.

Summarizing the above discussion, we just need to consider a situation when $\operatorname{dim}\left(\operatorname{Lin}_{\mathbb{Q}}\left(x_{1}^{\xi}, x_{2}^{\xi}, x_{3}^{\xi}\right)\right)=2$ and $\operatorname{Lin}_{\mathbb{Q}}\left(x_{1}^{\xi}, x_{2}^{\xi}, x_{3}^{\xi}\right) \cap \operatorname{Lin}_{\mathbb{Q}}(X)=\{0\}$ for all $\xi$. Recall that $q_{1} x_{1}^{\xi}+q_{2} x_{2}^{\xi}+q_{3} x_{3}^{\xi}=0$, where $q_{1}, q_{2}, q_{3} \in \mathbb{Q} \backslash\{0\}$. If two of $x_{1}^{\xi}, x_{2}^{\xi}, x_{3}^{\xi}$ were dependent over $\mathbb{Q}$, then we would have $\operatorname{dim}\left(\operatorname{Lin}_{\mathbb{Q}}\left(x_{1}^{\xi}, x_{2}^{\xi}, x_{3}^{\xi}\right)\right) \leq 1$. Thus, $x_{1}^{\xi}, x_{2}^{\xi}, x_{3}^{\xi}$ are pairwise independent. Now it is easy to see that case (b) holds.

Lemma 3.8. Let $X \in\left[\mathbb{R}^{n}\right]^{<\mathfrak{c},} x \notin X$, and $y \in \mathbb{R}$. Suppose also that $h, g: X \rightarrow \mathbb{R}$ are functions linearly independent over $\mathbb{Q}$. Then there exist extensions $h^{\prime}, g^{\prime}$ of $h$ and $g$ onto $X \cup\{x\}$ such that $h^{\prime}$ and $g^{\prime}$ are linearly independent over $\mathbb{Q}$ and $h^{\prime}(x)+g^{\prime}(x)=y$.

Proof. Choose $h^{\prime}(x) \in \mathbb{R} \backslash \operatorname{Lin}_{\mathbb{Q}}(h[X] \cup g[X] \cup\{y\})$. This choice is possible since $\left|\operatorname{Lin}_{\mathbb{Q}}(h[X] \cup g[X] \cup\{y\})\right|<\mathfrak{c}$. Then define $g^{\prime}(x)=y-h^{\prime}(x)$. It is easy to see that $h^{\prime}=h \cup\left\{\left\langle x, h^{\prime}(x)\right\rangle\right\}$ and $g^{\prime}=g \cup\left\{\left\langle x, g^{\prime}(x)\right\rangle\right\}$ are the desired extensions.

Proof of Theorem 3.4 Let us start by fixing a function $f: \mathbb{R}^{n} \rightarrow \mathbb{R}$ and enumerations $\left\{x_{\xi}: \xi<\mathfrak{c}\right\},\left\{v_{\xi}: \xi<\mathfrak{c}\right\}$ of $\mathbb{R}^{n}$ and $\{0\} \times \mathbb{R} \subseteq \mathbb{R}^{n+1}$, respectively. We will construct functions $h, g: \mathbb{R}^{n} \rightarrow \mathbb{R}$ which are linearly independent over $\mathbb{Q}$ and satisfy the property that $h+g=f$ and $\{0\} \times \mathbb{R} \subseteq \operatorname{Lin}_{\mathbb{Q}}(h) \cap \operatorname{Lin}_{\mathbb{Q}}(g)$.

First, let us argue that this is enough to prove the theorem. What we have to show is that $\operatorname{Lin}_{\mathbb{Q}}(h)=\operatorname{Lin}_{\mathbb{Q}}(g)=\mathbb{R}^{n+1}$. To see $\operatorname{Lin}_{\mathbb{Q}}(h)=\mathbb{R}^{n+1}$ note that

$\forall x \in \mathbb{R}^{n} \forall z \in \mathbb{R}\langle x, z\rangle=\langle x, h(x)\rangle+\langle 0, z-h(x)\rangle \in \operatorname{Lin}_{\mathbb{Q}}(h)+\operatorname{Lin}_{\mathbb{Q}}(h)=\operatorname{Lin}_{\mathbb{Q}}(h)$.

By the same argument $\operatorname{Lin}_{\mathbb{Q}}(g)=\mathbb{R}^{n+1}$.

To construct the desired functions $h$ and $g$, we consider three cases. In the first case we assume that $|\mathrm{LC}(f)|<\mathfrak{c}$. If the latter fails, that is $|\mathrm{LC}(f)|=\mathfrak{c}$, then either part (a) (Case 2) or part (b) (Case 3) of Property [3.7]holds.

Case 1: $|\mathrm{LC}(f)|<\mathfrak{c}$.

Let $\kappa<\mathfrak{c}$ denote the cardinality of the basis of $\operatorname{LC}(f)$ over $\mathbb{Q}$. There exist $c \in \mathrm{LC}(f)$ and a linearly independent set $A \subseteq \mathbb{R}^{n}$ such that $|A|=\kappa$ and $f(-a)+$ $f(a) \equiv c=$ const for all $a \in A$. Such a set can be found since $|\operatorname{LC}(f)|<\mathfrak{c}$ and $f(x)+f(-x) \in \operatorname{LC}(f)$ for every $x \in \mathbb{R}^{n}$. Put $B=(-A) \cup A$.

First, we will construct functions $h, g: B \rightarrow \mathbb{R}$ linearly independent over $\mathbb{Q}$ for which $h+g \subseteq f$ and

$$
\{0\} \times \operatorname{LC}(f) \subseteq \operatorname{Lin}_{\mathbb{Q}}(h) \cap(\{0\} \times \mathbb{R})=\operatorname{Lin}_{\mathbb{Q}}(g) \cap(\{0\} \times \mathbb{R}) .
$$


To accomplish this let us fix enumerations $\left\{a_{\xi}: \xi<\kappa\right\}$ of $A$ and $\left\{m_{\xi}: \xi<\kappa\right\}$ of a linear basis of $\operatorname{LC}(f)$ over $\mathbb{Q}$. We may assume that $m_{0}=c$ if $c \neq 0$. The construction of $h$ and $g$ is by induction. At every step $\alpha<\kappa$ we will define $h$ and $g$ on $\left\{-a_{\alpha}, a_{\alpha}\right\}$, assuring that

(a) $h\left|A_{\alpha}, g\right| A_{\alpha}$ are linearly independent and $(h+g) \mid A_{\alpha} \subseteq f$,

(b) $\left\langle 0, m_{\alpha}\right\rangle \in \operatorname{Lin}_{\mathbb{Q}}\left(h \mid A_{\alpha}\right) \cap(\{0\} \times \mathbb{R})=\operatorname{Lin}_{\mathbb{Q}}\left(g \mid A_{\alpha}\right) \cap(\{0\} \times \mathbb{R})$,

where $A_{\alpha}=\left\{i a_{\xi}: \xi \leq \alpha, i=-1,1\right\}$.

For $\alpha=0$ and $x= \pm a_{0}$ put $h(x)=\frac{1}{4} m_{0}$ and $g(x)=f(x)-h(x)$. Observe that $g\left(-a_{0}\right)+g\left(a_{0}\right)=\left[f\left(-a_{0}\right)+f\left(a_{0}\right)\right]-\left[h\left(-a_{0}\right)+h\left(a_{0}\right)\right] \in\left\{-\frac{1}{2} m_{0}, \frac{1}{2} m_{0}\right\}$. This holds because $f\left(-a_{0}\right)+f\left(a_{0}\right)=c$ and $m_{0}=c$ if $c \neq 0$. Thus, $\langle 0, c\rangle,\left\langle 0, m_{0}\right\rangle \in$ $\operatorname{Lin}_{\mathbb{Q}}\left(h \mid A_{0}\right) \cap \operatorname{Lin}_{\mathbb{Q}}\left(g \mid A_{0}\right)$. It is easily seen that $h \mid A_{0}$ and $g \mid A_{0}$ satisfy (a) and (b).

Now suppose that $h$ and $g$ are defined on $A_{<\alpha}=\bigcup_{\xi<\alpha} A_{\xi}, \alpha<\kappa$, and they satisfy conditions (a) and (b) for all $\xi<\alpha$. We will extend $h$ and $g$ onto $A_{\alpha}$, preserving the desired properties.

We may assume that $\left\langle 0, m_{\alpha}\right\rangle \notin \operatorname{Lin}_{\mathbb{Q}}\left(h \mid A_{<\alpha}\right) \cup \operatorname{Lin}_{\mathbb{Q}}\left(g \mid A_{<\alpha}\right)$. (Otherwise we could extend $h$ and $g$ using Lemma 3.8, preserving the condition (a).) Put $h(x)=\frac{1}{2} m_{\alpha}$ and $g(x)=f(x)-h(x)$ for $x \in\left\{-a_{\alpha}, a_{\alpha}\right\}$. We claim that (a) and (b) are satisfied. Obviously, $(h+g) \mid A_{\alpha} \subseteq f$. To see the linear independence of $h \mid A_{\alpha}$ and $g \mid A_{\alpha}$ first note that, based on the inductive assumption, $h \mid A_{<\alpha}$ and $g \mid A_{<\alpha}$ are linearly independent. Next suppose that

$$
p\left\langle-a_{\alpha}, h\left(-a_{\alpha}\right)\right\rangle+q\left\langle a_{\alpha}, h\left(a_{\alpha}\right)\right\rangle=v \text { for some } p, q \in \mathbb{Q} \text { and } v \in \operatorname{Lin}_{\mathbb{Q}}\left(h \mid A_{<\alpha}\right) .
$$

Since $a_{\alpha} \notin \operatorname{Lin}_{\mathbb{Q}}\left(A_{<\alpha}\right)$ we conclude that $p=q$. Therefore we have

$$
p\left\langle-a_{\alpha}, h\left(-a_{\alpha}\right)\right\rangle+q\left\langle a_{\alpha}, h\left(a_{\alpha}\right)\right\rangle=p\left\langle 0, h\left(-a_{\alpha}\right)+h\left(a_{\alpha}\right)\right\rangle=p\left\langle 0, m_{\alpha}\right\rangle=v .
$$

But we assumed that $\left\langle 0, m_{\alpha}\right\rangle \notin \operatorname{Lin}_{\mathbb{Q}}\left(h \mid A_{<\alpha}\right) \cup \operatorname{Lin}_{\mathbb{Q}}\left(g \mid A_{<\alpha}\right)$, so $p=0$ and $v=0$. This shows the linear independence of $h \mid A_{\alpha}$. A very similar argument works for $g \mid A_{\alpha}$ : just notice that $g\left(-a_{\alpha}\right)+g\left(a_{\alpha}\right)=\left[f\left(-a_{\alpha}\right)+f\left(a_{\alpha}\right)\right]-\left[h\left(-a_{\alpha}\right)+h\left(a_{\alpha}\right)\right]=$ $c-m_{\alpha}$ and recall that $\langle 0, c\rangle \in \operatorname{Lin}_{\mathbb{Q}}\left(g \mid A_{0}\right) \subseteq \operatorname{Lin}_{\mathbb{Q}}\left(g \mid A_{<\alpha}\right)$.

Now we show that (b) is also satisfied. From what has already been proved, we conclude that $\left\langle 0, m_{\alpha}\right\rangle \in \operatorname{Lin}_{\mathbb{Q}}\left(h \mid A_{\alpha}\right) \cup \operatorname{Lin}_{\mathbb{Q}}\left(g \mid A_{\alpha}\right)$.

Thus, what remains to prove is the equality part of (b). (The following argument is also needed in the case when Lemma 3.8 was used to define $h$ and $g$ on $\left\{-a_{\alpha}, a_{\alpha}\right\}$.) It follows from the fact that $\langle 0, y\rangle \in \operatorname{Lin}_{\mathbb{Q}}\left(h \mid A_{\alpha}\right)$, provided there exist $p_{i} \in \mathbb{Q}$ and $a_{i} \in A_{\alpha}, i \leq n$, such that

$$
\begin{aligned}
\langle 0, y\rangle & =\sum_{1}^{m} p_{i}\left[\left\langle-a_{i}, h\left(-a_{i}\right)\right\rangle+\left\langle a_{i}, h\left(a_{i}\right)\right\rangle\right] \\
& =\sum_{1}^{m} p_{i}\left\langle 0, h\left(-a_{i}\right)+h\left(a_{i}\right)\right\rangle \\
& =\sum_{1}^{m} p_{i}\left\langle 0, f\left(-a_{i}\right)+f\left(a_{i}\right)\right\rangle-p_{i}\left\langle 0, g\left(-a_{i}\right)+g\left(a_{i}\right)\right\rangle \\
& =\sum_{1}^{m} p_{i}\langle 0, c\rangle-\sum_{1}^{m} p_{i}\left[\left\langle-a_{i}, g\left(-a_{i}\right)\right\rangle+\left\langle a_{i}, g\left(a_{i}\right)\right\rangle\right] \\
& \in \operatorname{Lin}_{\mathbb{Q}}\left(g \mid A_{\alpha}\right) .
\end{aligned}
$$


This completes the inductive definition of $h$ and $g$. Note that (3.3) implies that any extensions $h^{\prime}, g^{\prime}$ of $h$ and $g$, with $h^{\prime}+g^{\prime} \subseteq f$, also satisfy

$$
\{0\} \times \operatorname{LC}(f) \subseteq \operatorname{Lin}_{\mathbb{Q}}\left(h^{\prime}\right) \cap(\{0\} \times \mathbb{R})=\operatorname{Lin}_{\mathbb{Q}}\left(g^{\prime}\right) \cap(\{0\} \times \mathbb{R}) .
$$

To see this choose $\langle 0, y\rangle \in \operatorname{Lin}_{\mathbb{Q}}\left(h^{\prime}\right) \cap(\{0\} \times \mathbb{R})$. So for some $p_{i} \in \mathbb{Q}$ and $x_{i} \in$ $\mathbb{R}^{n}$ we have $\langle 0, y\rangle=\sum_{1}^{m} p_{i}\left\langle x_{i}, h^{\prime}\left(x_{i}\right)\right\rangle=\sum_{1}^{m} p_{i}\left\langle x_{i}, f\left(x_{i}\right)\right\rangle-\sum_{1}^{m} p_{i}\left\langle x_{i}, g^{\prime}\left(x_{i}\right)\right\rangle \in$ $\operatorname{Lin}_{\mathbb{Q}}\left(g^{\prime}\right) \cap(\{0\} \times \mathbb{R})$. The latter holds because $\sum_{1}^{m} p_{i} x_{i}=0$ and consequently $\sum_{1}^{m} p_{i} f\left(x_{i}\right) \in \mathrm{LC}(f)$. This ends the proof of 3.4 .

Next we extend $h$ and $g$ onto $\mathbb{R}^{n}=\left\{x_{\xi}: \xi<\mathfrak{c}\right\}$, preserving the linear independence and the property that at a step $\xi$ of the inductive definition we assure that $x_{\xi} \in \operatorname{dom}\left(h_{\xi}\right)=\operatorname{dom}\left(g_{\xi}\right)$ and $v_{\xi} \in \operatorname{Lin}_{\mathbb{Q}}\left(h_{\xi}\right) \cap \operatorname{Lin}_{\mathbb{Q}}\left(g_{\xi}\right)$, where $h_{\xi}$ and $g_{\xi}$ denote the extensions obtained in the step $\xi$.

Let $\beta<\mathfrak{c}$. Assume that $v_{\beta} \notin \operatorname{Lin}_{\mathbb{Q}}\left(\bigcup_{\xi<\beta} h_{\xi}\right) \cup \operatorname{Lin}_{\mathbb{Q}}\left(\bigcup_{\xi<\beta} g_{\xi}\right)$. Choose an $a \in \mathbb{R} \backslash \operatorname{Lin}_{\mathbb{Q}}\left(\operatorname{dom}\left(\bigcup_{\xi<\beta} h_{\xi}\right)\right)$ and define $h(x)$ by $\langle 0, h(x)\rangle=\frac{1}{2} v_{\beta}$ for $x \in\{-a, a\}$. Also put $g(x)=f(x)-h(x)$. Since $f(-a)+f(a) \in \mathrm{LC}(f)$, (3.3) implies that $v_{\beta} \in \operatorname{Lin}_{\mathbb{Q}}(h) \cap \operatorname{Lin}_{\mathbb{Q}}(g)$. What remains to show is that $h$ and $g$ are still linearly independent. But this follows from (3.4) and almost the same argument which is used to show the linear independence of $h \mid A_{\alpha}$ and $g \mid A_{\alpha}$ in the previous part of the proof. (Replace $a_{\alpha}, h \mid A_{<\alpha}$, and $g \mid A_{<\alpha}$ by $a, \bigcup_{\xi<\beta} h_{\xi}$, and $\bigcup_{\xi<\beta} g_{\xi}$, respectively.)

To finish step $\beta$ of the inductive definition we need to make sure that $h$ and $g$ are defined at $x_{\beta}$. If $x_{\beta} \notin \operatorname{dom}(h)=\operatorname{dom}(g)$, then we can use Lemma 3.8 to define these functions at $x_{\beta}$, preserving all the required properties. This ends the construction in Case 1.

Case 2: Property [3.7(a) holds.

Let $Z \in\left[\mathbb{R}^{n}\right]^{<\mathfrak{c}}$ be a set satisfying $\left|\bigcup_{z \in Z} \mathrm{LC}(f, 2, z)\right|=\mathfrak{c}$. We start by defining functions $h, g: Z \rightarrow \mathbb{R}$ which are linearly independent over $\mathbb{Q}$ and whose sum is contained in $f$ (i.e., $h+g \subseteq f$ ). It can be easily done by using Lemma 3.8

We will extend $h$ and $g$ onto $\mathbb{R}^{n}$ by induction. Let $\beta<\mathfrak{c}$. Assume that $h$ and $g$ are linearly independent, $h+g \subseteq f,\left\{x_{\xi}: \xi<\beta\right\} \subseteq D_{\beta}=\operatorname{dom}(h)=\operatorname{dom}(g)$, $\left\{v_{\xi}: \xi<\beta\right\} \subseteq \operatorname{Lin}_{\mathbb{Q}}(h) \cap \operatorname{Lin}_{\mathbb{Q}}(g)$, and $v_{\beta} \notin \operatorname{Lin}_{\mathbb{Q}}(h)$. The property of the set $Z$ implies the existence of a $z \in Z$ satisfying $|\mathrm{LC}(f, 2, z)|>\max (|h|, \omega)=\max (|g|, \omega)$. Thus, an easy cardinal argument shows that we can find $z_{1}, z_{2} \in \mathbb{R}^{n} \backslash \operatorname{Lin}_{\mathbb{Q}}\left(D_{\beta}\right)$ and $p_{1}, p_{2} \in \mathbb{Q} \backslash\{0\}$ which satisfy

$$
p_{1} z_{1}+p_{2} z_{2}=z \text { and }\left\langle z, p_{1} f\left(z_{1}\right)+p_{2} f\left(z_{2}\right)\right\rangle \notin \operatorname{Lin}_{\mathbb{Q}}\left(g \cup\left\{\langle 0, h(z)\rangle, v_{\beta}\right\}\right) .
$$

Define the values of $h$ at $z_{1}$ and $z_{2}$ so that

$$
p_{1}\left\langle z_{1}, h\left(z_{1}\right)\right\rangle+p_{2}\left\langle z_{2}, h\left(z_{2}\right)\right\rangle=\left\langle z, p_{1} h\left(z_{1}\right)+p_{2} h\left(z_{2}\right)\right\rangle=v_{\beta}+\langle z, h(z)\rangle .
$$

Observe that $v_{\beta}=\left[v_{\beta}+\langle z, h(z)\rangle\right]-\langle z, h(z)\rangle \in \operatorname{Lin}_{\mathbb{Q}}(h)$.

Now we argue that $h$ and $g$ are linearly independent. To see the linear independence of $h$ suppose that for some $q, r \in \mathbb{Q}$ (not both equal 0 ) we have

$$
q\left\langle z_{1}, h\left(z_{1}\right)\right\rangle+r\left\langle z_{2}, h\left(z_{2}\right)\right\rangle=\left\langle q z_{1}+r z_{2}, q h\left(z_{1}\right)+r h\left(z_{2}\right)\right\rangle \in \operatorname{Lin}_{\mathbb{Q}}\left(h \mid D_{\beta}\right) .
$$

Since $z_{1}, z_{2} \notin \operatorname{Lin}_{\mathbb{Q}}\left(D_{\beta}\right)$ and $p_{1} z_{1}+p_{2} z_{2}=z \in Z \subseteq \operatorname{Lin}_{\mathbb{Q}}\left(D_{\beta}\right)$ we conclude that $\langle q, r\rangle$ and $\left\langle p_{1}, p_{2}\right\rangle$ are linearly dependent. So we may assume that $\langle q, r\rangle=\left\langle p_{1}, p_{2}\right\rangle$. Consequently, $v_{\beta}+\langle z, h(z)\rangle=\left\langle z, p_{1} h\left(z_{1}\right)+p_{2} h\left(z_{2}\right)\right\rangle \in \operatorname{Lin}_{\mathbb{Q}}\left(h \mid D_{\beta}\right)$. This contradicts the assumption $v_{\beta} \notin \operatorname{Lin}_{\mathbb{Q}}\left(h \mid D_{\beta}\right)$. Hence, $h$ is linearly independent. 
Based on the above argument, we see that the linear independence of $g$ will follow from $\left\langle z, p_{1} g\left(z_{1}\right)+p_{2} g\left(z_{2}\right)\right\rangle \notin \operatorname{Lin}_{\mathbb{Q}}\left(g \mid D_{\beta}\right)$. But this holds since (3.5) implies

$$
\begin{aligned}
\left\langle z, p_{1} g\left(z_{1}\right)+p_{2} g\left(z_{2}\right)\right\rangle & \\
\left\langle z, p_{1} f\left(z_{1}\right)+p_{2} f\left(z_{2}\right)-\left[p_{1} h\left(z_{1}\right)+p_{2} h\left(z_{2}\right)\right]\right\rangle & = \\
\left\langle z, p_{1} f\left(z_{1}\right)+p_{2} f\left(z_{2}\right)\right\rangle-\langle 0, h(z)\rangle-v_{\beta} & \notin \operatorname{Lin}_{\mathbb{Q}}\left(g \mid D_{\beta}\right) .
\end{aligned}
$$

To assure that $v_{\beta} \in \operatorname{Lin}_{\mathbb{Q}}(g)$ we repeat the same procedure as above for the function $g$. Finally, if $x_{\beta} \notin \operatorname{dom}(h)=\operatorname{dom}(g)$, then we use Lemma 3.8 to define the functions at $x_{\beta}$. This ends the construction in Case 2 .

Case 3: Property [3.7(b) holds.

The inductive construction of functions $h$ and $g$ is somewhat similar to the one in the previous case. So assume that $\beta<\mathfrak{c}$ and the construction has been carried out for all $\xi<\beta$. If $v_{\beta} \notin \operatorname{Lin}_{\mathbb{Q}}(h)$, then let $X=\operatorname{dom}(h)=\operatorname{dom}(g)$ and $Y \in[\mathbb{R}]^{<\mathfrak{c}}$ be such a set that $\operatorname{Lin}_{\mathbb{Q}}\left(g \cup\left\{v_{\beta}\right\}\right) \subseteq \mathbb{R}^{n} \times Y$. By Property [3.7(b), there exist $p_{1}, p_{2}, p_{3} \in \mathbb{Q} \backslash\{0\}$ and pairwise independent $x_{1}, x_{2}, x_{3} \in \mathbb{R}^{n}$ such that $\sum_{1}^{3} p_{i} x_{i}=0$, $\operatorname{Lin}_{\mathbb{Q}}\left(x_{1}, x_{2}, x_{3}\right) \cap \operatorname{Lin}_{\mathbb{Q}}(X)=\{0\}$, and $\sum_{1}^{3} p_{i} f\left(x_{i}\right) \notin Y$.

We extend $h$ and $g$ onto $\left\{x_{1}, x_{2}, x_{3}\right\}$. Choose $h\left(x_{1}\right), h\left(x_{2}\right), h\left(x_{3}\right) \in \mathbb{R}$ in such a way that

$$
\sum_{1}^{3} p_{i}\left\langle x_{i}, h\left(x_{i}\right)\right\rangle=\left\langle 0, \sum_{1}^{3} p_{i} h\left(x_{i}\right)\right\rangle=v_{\beta} .
$$

Then put $g\left(x_{i}\right)=f\left(x_{i}\right)-h\left(x_{i}\right)$ for $i \leq 3$. Obviously $v_{\beta} \in \operatorname{Lin}_{\mathbb{Q}}(h)$ and $h+g \subseteq f$. We claim that the linear independence of $h$ and $g$ is also preserved.

To show this claim note first that, if $\sum_{1}^{3} p_{i}^{\prime} x_{i} \in \operatorname{Lin}_{\mathbb{Q}}(X)$ for some $p_{1}^{\prime}, p_{2}^{\prime}, p_{3}^{\prime} \in \mathbb{Q}$, then $\sum_{1}^{3} p_{i}^{\prime} x_{i}=0$. Pairwise independence of $x_{1}, x_{2}, x_{3}$ implies that $\sum_{1}^{3} p_{i}^{\prime} x_{i}=0$ holds only for triples $\left\langle p_{1}^{\prime}, p_{2}^{\prime}, p_{3}^{\prime}\right\rangle \in \operatorname{Lin}_{\mathbb{Q}}\left(\left\langle p_{1}, p_{2}, p_{3}\right\rangle\right)$. Thus, our claim holds if $\sum_{1}^{3} p_{i}\left\langle x_{i}, h\left(x_{i}\right)\right\rangle \notin \operatorname{Lin}_{\mathbb{Q}}(h \mid X)$ and $\sum_{1}^{3} p_{i}\left\langle x_{i}, g\left(x_{i}\right)\right\rangle \notin \operatorname{Lin}_{\mathbb{Q}}(g \mid X)$. But these two conditions follow from

- $\sum_{1}^{3} p_{i}\left\langle x_{i}, h\left(x_{i}\right)\right\rangle=v_{\beta} \notin \operatorname{Lin}_{\mathbb{Q}}(h \mid X)$ and

- $\sum_{1}^{3} p_{i}\left\langle x_{i}, g\left(x_{i}\right)\right\rangle=\sum_{1}^{3} p_{i}\left\langle x_{i}, f\left(x_{i}\right)-h\left(x_{i}\right)\right\rangle=\left\langle 0, \sum_{1}^{3} p_{i} f\left(x_{i}\right)\right\rangle-v_{\beta} \notin \operatorname{Lin}_{\mathbb{Q}}(g \mid X)$.

(The " $\notin$ " part holds because $\operatorname{Lin}_{\mathbb{Q}}\left((g \mid X) \cup\left\{v_{\beta}\right\}\right) \subseteq \mathbb{R}^{n} \times Y$ and $\sum_{1}^{3} p_{i} f\left(x_{i}\right) \notin Y$.)

To assure that $v_{\beta} \in \operatorname{Lin}_{\mathbb{Q}}(g)$ we repeat the same steps as above for the function $g$ and then, if necessary, define $h$ and $g$ at $x_{\beta}$ using Lemma 3.8. This ends the construction in Case 3.

\section{REFERENCES}

[1] A.L Cauchy, Cours d'analyse de l'Ecole Polytechnique, 1. Analyse algébrique, V., Paris, 1821 [Oeuvres (2) 3, Paris, 1897]. MR 95a:01026

[2] K. Ciesielski, Set Theory for the Working Mathematician, London Math. Soc. Student Texts 39, Cambridge Univ. Press 1997. MR 99c:04001

[3] K. Ciesielski, J. Jastrzębski, Darboux-like functions within the classes of Baire one, Baire two, and additive functions, Topology Appl. 103 (2000), 203-219. MR 2001g:26001

[4] K. Ciesielski, T. Natkaniec, Algebraic properties of the class of Sierpinski-Zygmund functions, Topology Appl. 79 (1997), 75-99. MR 99c:04003

[5] K. Ciesielski, I. Recław, Cardinal invariants concerning extendable and peripherally continuous functions, Real Anal. Exchange 21 (1995-96), 459-472. MR 97f:26003

[6] G. Hamel, Eine Basis aller Zahlen und die unstetigen Lösungen der Funktionalgleichung $f(x+y)=f(x)+f(y)$, Math. Ann. 60 (1905), 459-462. 
[7] W. Hurewicz and H. Wallman, Dimension Theory, Princeton University Press, 1941. MR 3:312b

[8] M. Kuczma, An Introduction to the Theory of Functional Equations and Inequalities, Polish Scientific Publishers, PWN, Warszawa, 1985. MR 86i:39008

[9] C. Kuratowski, Topologie I, Warszawa 1958. MR 19:873d

[10] T. Natkaniec, Almost Continuity, Real Anal. Exchange 17 (1991-92), 462-520. MR 93e:54009

[11] J.C. Oxtoby, Measure and Category, Springer-Verlag, Amsterdam 1971. MR 52:14213

[12] K. Płotka, Sum of Sierpiński-Zygmund and Darboux Like functions, Topology Appl. 122/3 (2002), 547-564.

Department of Mathematics, University of Scranton, Scranton, Pennsylvania 18510

E-mail address: plotkak2@scranton.edu 